\title{
Valutazione di un test per la determinazione simultanea degli anticorpi e antigene p24 dell'HIV
}

\author{
Nadia Zanchetta, Carla Bossi, Claudia Tonielli, Claudio Galli' \\ U. O. Microbiologia, Azienda Ospedaliera Polo Universitario L. Sacco Milano \\ ' Medical Marketing, Abbott Divisione Diagnostici, Roma
}

Key words: HIV, 4th generation assay, p24, anti-HIV, screening

Evaluation of an assay for the simultaneous detection of anti-HIV antibodies and p24 antigen

\section{SUMMARY}

A fourth generation immunoassay for the detection of antibodies to HIV-I and HIV-2 and of HIV antigen p24 (Abbott Architect HIV Ag/Ab Combo) has been evaluated in comparison with two Ab-only third generation assays on 894 routine specimens and on preselected repository specimens. The Combo assay showed a better specificity (99.88\% vs. $99.43-99.83 \%$ ) and an analytical sensitivity for p24 of $22 \mathrm{pg} / \mathrm{mL}$ on a BBI commercial panel. The Architect assays gave a negative result on 22/24 repository false positive specimens from 18 subjects and, conversely, was positive on all the 39 repository specimens from $24 \mathrm{HIV}$-positive patients. On six patients with acute HIV infection the Architect assay gave an earlier positivity than the antibody-only assays (EIA and WB) on three cases, all viremic and positive for HIV p24. The performance characteristics of the new HIV Combo assay guarantee an advanced clinical sensitivity and a high specificity.

\section{INTRODUZIONE}

L'andamento nel tempo dei casi di AIDS in Italia, notificati al Registro Nazionale AIDS (8), è sempre stato in crescita sino al 1995; dopo tale data vi è stata una considerevole diminuzione dei casi notificati (tra il 1996 e 2001), con una successiva stabilità che permane tutt'ora. I tassi di incidenza dimostrano che le regioni più colpite sono, nell'ordine, Lombardia, Lazio, Liguria ed Emilia Romagna, evidenziando un maggior interessamento delle regioni del nord rispetto al sud. Sono in aumento i casi di AIDS tra i cittadini stranieri, dal 4,5\% notificati nel 1994 al 17,5\% nel 2004, e il $69,7 \%$ dei casi totali di AIDS sono concentrati nella fascia di età tra i 25-39 anni, con un progressivo maggior coinvolgimento del sesso femminile (7,2 \% nel 1997, 31,7\% nel 2002). Inoltre si è spostata l'età media di diagnosi tra gli adulti che nel 1985 era di 29 anni per i maschi e 24 per le femmine ed è diventata nel 2004 rispettivamente di 41 e 38 anni. Le modalità di trasmissione in quasi il $60 \%$ dei casi tra gli adulti è riconducibile a pratiche sessuali (sia omo che eterosessuali).

Nel versante pediatrico, la diffusione e l'andamento dell'AIDS sembrano ricalcare geograficamente l'andamento degli adulti. La significativa diminuzione dei casi pediatrici, a partire dal 1997 , è riconducibile primariamente al trattamento antiretrovirale delle donne sieropositive e in gravidanza, al fine di ridurre la trasmissione verticale, e secondariamente all'effetto della terapia antiretrovirale nei bambini.

Il sistema di sorveglianza dei casi di AIDS, in Italia ha costituito, sino ad ora un valido mezzo per acquisire informazioni su questa epidemia; come l'andamento nel tempo o i cambiamenti della popolazione coinvolta. La terapia antiretrovirale combinata (HAART) ha portato delle modifiche quali l'allungamento della sopravvivenza, il miglioramento della qualità della vita e la modifica del periodo di incubazione della malattia (1). Fermo restando ciò, le notifiche dei casi di AIDS non costituiscono più una stima adeguata dell'incidenza e prevalenza dell'infezione da HIV nel nostro paese. Risulta quindi importante che l'andamento di questa epidemia venga monitorato sullo studio delle infezioni recenti, per poter predisporre interventi mirati alla prevenzione. La raccolta dei dati in tal senso è già iniziata in alcune regioni pilota, e ha permesso di evidenziare un andamento per certi versi simile a quello dei casi di AIDS. Dopo un picco all'inizo degli anni '90 si è infatti assistito ad una diminuzione dei nuovi casi di infezione da HIV, che è stata però seguita da una nuova crescita negli ultimi due-tre anni. I nuovi infetti sono prevalentemente adulti che acquisiscono l'infezione per via sessuale, appartenenti quindi a categorie a rischio diverse da quelle degli anni '80. Inoltre questi dati se pur parziali, non essendo ancora attiva una sorveglianza nazionale delle infezioni da HIV, consentono di 
stimare un'incidenza (nuovi casi di infezione) tra 4 e 10 per 100.000 abitanti/anno (7).

In questo contesto risulta importante poter disporre di mezzi di prevenzione in grado di diagnosticare precocemente le nuove infezioni, caratterizzati quindi da un'elevata sensibilità e in grado di evidenziare nel minor tempo possibile i nuovi casi. Considerando quindi la necessità e utilità di avere a disposizione test di screening che consentano una precoce evidenziazione dell'infezione da HIV, è stato valutato un test di "quarta generazione" in chemiluminescenza, (Abbott Architect $\mathrm{HIV} \mathrm{Ag/Ab} \mathrm{Combo)} \mathrm{per} \mathrm{la} \mathrm{determinazione} \mathrm{simul-}$ tanea dell'Antigene p24 (Agp24) e degli anticorpi anti-HIV 1-2, in confronto con due test di terza generazione (Abbott AxSYM HIV 1-2gO e AntiHIV Tetra-Biotest) in diverse tipologie di popolazione a rischio e non per l'infezione da HIV.

\section{MATERIALI E METODI}

Sono stati analizzati 301 campioni di siero ottenuti da donatori di sangue; rispettivamente 219 maschi (M) (età media 42,7 $\pm 12,1$ anni) e 82 femmine $(\mathrm{F})$ (età media 40,7 $\pm 12,9$ anni), e 593 campioni di siero della routine diagnostica del nostro laboratorio ottenuti da pazienti ricoverati o ambulatoriali, rispettivamente 213 (M) (età media 45,1 $\pm 18,0 \mathrm{a}), 331 \mathrm{~F}$ ) (età media $39,5 \pm 16,6$ anni) e 49 pazienti dei quali non era noto il sesso (età media $37,7+9,7$ anni).

Abbiamo inoltre preso in considerazione 57 campioni provenienti da una sieroteca, appartenenti a 42 pazienti rispettivamente $14(\mathrm{~F})$ e $28(\mathrm{M})$ di età compresa tra 1 mese e 70 anni, risultati discordanti alle diverse metodiche sierologiche e di biologia molecolare per HIV, così classificati: A) 18 pazienti HIV-negativi; B) 20 pazienti HIV-positivi; C) 4 bambini di età inferiore ai 2 a ( $2 \mathrm{M}$ e 2 F), nati da madre HIV positiva; dei quali 3 HIVnegativi e 1 HIV-positivo.

Infine abbiamo considerato 6 sieroconversioni recenti ( $1 \mathrm{~F}$ e $5 \mathrm{M}$, età compresa tra 19 e 75 anni), seguite nel tempo, con test sierologici e di biologia molecolare, diagnosticate nell'arco di 3 mesi (da settembre a novembre 2005).

La valutazione è stata condotta analizzando i campioni della routine diagnostica in parallelo con il test Architect e con i due test per soli anticorpi anti-HIV, di terza generazione: AxSYM HIV 1-2gO e anti-HIV Tetra-Biotest. Tutti i campioni della routine risultati positivi o "borderline" con uno o più test di screening sono stati confermati mediante Western blot (WB); dove possibile, sono stati eseguiti anche un test per il solo Agp24 e/o la ricerca di HIV-DNA o HIV-RNA. I campioni di donatori di sangue sono stati analizzati in parallelo solamente con i test Architect e AxSYM.
I risultati ottenuti con i test di screening sono stati valutati mediante tabelle di contingenza allo scopo di valutare la concordanza e specificità relativa dei metodi.

Da un punto di vista analitico sono state valutate la riproducibilità nel tempo dei controlli interni del test Architect e di un controllo di qualità utilizzato nella routine del laboratorio. La linearità e sensibilità per Agp24 del test Architect è stata valutata utilizzando un pannello commerciale a concentrazioni scalari di Agp24 (BBI PRA 801).

\section{RISULTATI}

Dei 301 donatori di sangue: 298 sono risultati negativi con entrambi i test (Architect e Axsym); i restanti 3 donatori, erano negativi con Architect e positivi o "borderline" con AxSYM, con esito negativo in 2 casi e indeterminato (debole p24) in un caso alla WB. Quest'ultimo donatore era negativo tre mesi prima, mentre a due controlli successivi a distanza rispettivamente di 7 e 9 mesi dal campione risultato indeterminato alla WB era sempre presente una debole p24, con negatività sia per Agp24 che per e HIV-DNA.

Dei 593 campioni della routine diagnostica, 588 erano concordanti con tutti e tre $\mathrm{i}$ metodi (Architect, AxSYM e Biotest): 576 negativi e 12 positivi (1 campione positivo con tutti e tre $\mathrm{i}$ test era peraltro negativo alla WB, per HIV-DNA e per Agp24). Dei 5 campioni rimasti: 3 campioni erano positivi con AxSYM ma negativi in Architect, alla WB e per Agp24 e HIV-DNA, 1 campione (bambino di $16 \mathrm{~m}$ ), negativo in Architect e Biotest e per HIV-DNA, era "borderline" con AxSYM e indeterminato alla WB (debole p24); l'ultimo campione, era positivo in Architect e Biotest, "borderline" con AxSYM e indeterminato alla WB (debole p24), e non erano disponibili ulteriori controlli.

La concordanza complessiva tra Architect e AxSYM è stata del $99,32 \%$ e tra Architect e Biotest del 99,83\%. La specificità del test Architect è risultata del 99,88\%, per AxSYM del $99,43 \%$ e per Biotest $99,83 \%$ (figura I).

I controllo interni del test e del controllo di qualità interno del laboratorio (QI) sono stati analizzati in singolo in 21 sessioni nell'arco di 36 giorni. I coefficienti di variazione erano compresi tra $1 ' 8,8 \%$ ed il $16,9 \%$. In particolare, il controllo interno Virotrol ha presentato un CV totale del $12,1 \%$ con Architect e, nello stesso periodo, dell' $8,8 \%$ con il test AxSYM HIV1/2gO.

I risultati ottenuti con il test Architect sul pannello $\mathrm{BBI}$ hanno evidenziato una sensibilità pari a 22 $\mathrm{pg} / \mathrm{mL}$ (unità Pasteur) e $6 \mathrm{pg} / \mathrm{mL}$ (unità Dupont), un'ottima correlazione $(\mathrm{r}=0,992)$ e linearità $(r=0,999)$ con la concentrazione di p24 (figure II 
e III).

Sui 57 campioni di sieroteca relativi a 42 pazienti sono stati ottenuti i seguenti risultati:

a) Dei 18 pazienti classificati come negativi: 17 erano negativi in Architect; di questi erano 9 WB, 9 HIV-DNA, 4 Agp24 negativi, 8 WB indeterminate, 1 HIV-RNA $<50$ copie $/ \mathrm{ml}$; mentre solo un paziente è risultato positivo in Architect (figura IV) con valori "borderline" in AxSYM, indeterminato alla WB (p17) e negativo per Biotest e HIV-DNA.

b) I 20 pazienti classificati come positivi lo erano anche in Architect (esempio dei valori in figura V); 9/20 erano positivi in WB (6 HIV- DNA e 4 HIV-RNA positivi, 1 RNA $<50$ copie $/ \mathrm{ml}$ ); $11 / 20$ presentavano invece un "pattern" WB indeterminato (5 HIV-DNA e 8 HIV-RNA positivi).

c) 4 bambini di età inferiore ai 2 anni, erano positivi in Architect; 3 in fase di negativizzazione erano HIV-DNA negativi, un bambino era anche positivo in AxSYM, Biotest, WB e con HIV-RNA di $63.020 \mathrm{copie} / \mathrm{mL}$.

Le 6 sieroconversioni HIV, tutte diagnosticate tra settembre e novembre 2005, presentavano le seguenti caratteristiche:

Caso 1 (figura VI): donna africana di 27 anni, gravida alla $6^{\circ}$ settimana, riferiva una negatività per HIV nei due anni precedenti. Due mesi prima si era recata in Africa, al rientro episodio di Herpes genitale, con dolori addominali e neutropenia. Un primo test per HIV risultava positivo con il solo test Architect HIV Ag/Ab, negativo il test per soli anticorpi anti-HIV (Biotest) e alla WB, mentre sia il test per Ag p24 che quello per HIV-RNA erano positivi, rispettivamente $912 \mathrm{pg} / \mathrm{ml}$ e $>500.000$ copie $/ \mathrm{mL}$. Un controllo a distanza di una settimana presentava positività agli anticorpi (Biotest), WB indeterminata (p24) e Agp24 positivo (739 pg/mL). L'ultimo controllo a distanza di un mese dal primo, evidenziava l'avvenuta sieroconversione anche alla WB.

Caso 2 (figura VII): uomo di 35 anni, risultato negativo per anti-HIV due anni prima, con rapporti eterosessuali a rischio, a luglio del 2005 presenta febbre alternante con astenia, inappetenza e calo ponderale. Il paziente è stato ricoverato a fine agosto per candidosi (solo) esofagea con febbre alternante $\left(>39^{\circ} \mathrm{C}\right)$, mialgie, assenza di linfoadenopatia e interessamento alla milza. Ad un primo controllo per $\mathrm{HIV}$ risulta positivo per $\mathrm{HIV} \mathrm{Ag} / \mathrm{Ab}$ (Architect), debolmente positivo per soli anticorpi (Biotest), positivo per Ag p24 e HIV-RNA rispettivamente $1.437 \mathrm{pgr} / \mathrm{mL}$ e $>500.000 \mathrm{copie} / \mathrm{ml}$, negativo alla WB. Un controllo successivo a distanza di 13 giorni evidenziava una WB indeterminata (p24 e gp160), i due successivi controlli a distanza di un mese e mezzo e due mesi e mezzo dal primo presentavano un calo dell'HIV-RNA rispettivamente a 1.569 e 201 copie $/ \mathrm{mL}$.

Caso 3: uomo di 29 anni eterosessuale con rapporti a rischio, risultato sempre negativo per HIV negli anni precedenti, viene ricoverato per anemia aplastica, candidosi e ipertensione essenziale. Risulta positivo per HIV Ag/Ab (Architect) al primo test di screening, negativo per soli anticorpi (Biotest), positivo per Ag p24 $(793 \mathrm{pg} / \mathrm{mL})$ e HIV-RNA (> 500.000 copie $/ \mathrm{ml}$ ), indeterminato alla WB (debole p17 e p24). A poco più di un mese lo HIV-RNA scende a 1.735 copie $/ \mathrm{mL}$.

Caso 4: uomo di 19 anni, transessuale, arriva in pronto soccorso per sospetta tubercolosi con dolore toracico. La Mantoux e il PPD risultano negativi, al primo prelievo risulta positivo per $\mathrm{Ag} / \mathrm{Ab}$ (Architect) e negativo per soli anticorpi (Biotest), indeterminato alla WB (p24) positivo per Ag p24 e HIV-RNA rispettivamente 243 $\mathrm{pg} / \mathrm{mL}$ e $>500.000$ copie $/ \mathrm{mL}$. Ad un controllo dopo 15 giorni era riscontrata la positività agli anticorpi (Biotest) e alla WB, lo HIV-RNA era presente alla concentrazione di $4595 \mathrm{copie} / \mathrm{mL}$.

Gli ultimi due casi erano entrambi positivi sul primo prelievo sia per soli anticorpi (Biotest) che per $\mathrm{HIV} \mathrm{Ag} / \mathrm{Ab}$ (Architect) e indeterminati in WB (rispettivamente, deboli p24 e gp160 e deboli p24 gp120/160).

Il caso n.5 era un uomo di 32 anni, omosessuale, recatosi in pronto soccorso per astenia e febbre, il giorno successivo ricoverato in rianimazione per encefalite da HIV e mioclonie. Durante il ricovero vi è stato un episodio di polmonite da aspergillo, dopo un mese di terapia la carica virale di HIV-RNA era di 2.463 copie $/ \mathrm{mL}$ e, risolta la fase clinica acuta, il paziente è stato dimesso.

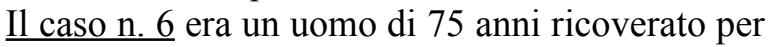
decadimento cognitivo e polineuropatia, l'RNA era al momento del ricovero 398.894 copie $/ \mathrm{mL}$, il valore calava a 53.730 dopo un mese.

\section{DISCUSSIONE}

La specificità pari a 99,88\% con il test Architect è risultata lievemente più elevata rispetto al valore dichiarato $(99,8 \%)$, probabilmente anche per l'entità numerica inferiore della casistica analizzata, ma comunque perfettamente all'interno dei limiti di confidenza del metodo. Il test combinato per HIV è risultato lievemente più specifico (figura I) rispetto ai due test per soli anticorpi analizzati in parallelo, confermando i dati di letteratura relativi ad un analogo test combinato su piattaforma $\operatorname{AxSYM}(5,6)$.

Considerando le sei sieroconversioni recenti si può notare che in tre casi la positività sul primo prelievo era rilevabile solo con il test Architect HIV Combo, e quattro casi erano negativi anche 
alla WB, mentre tutti risultavano positivi per HIV-RNA e Ag p24, se dosato. Questi dati evidenziano l'importanza di indagare contemporaneamente la presenza dell'antigene e degli anticorpi per poter individuare precocemente le sieroconversioni recenti (4), come recentemente dimostrato in uno studio condotto nel nord Europa (2). L'adeguatezza dei dosaggi combinati per una precoce identificazione della positività è essenzialmente legata alla sensibilità analitica per l'antigene $\mathrm{p} 24$, che si positivizza in media 5 giorni dopo l'RNA virale (3); nella nostra esperienza la sensibilità del test Architect HIV AgAb Combo è risultata pari a $22 \mathrm{pg} / \mathrm{mL}$, in linea con i risultati dichiarati e con i dati della letteratura relativi ai test di maggiore sensibilità $(4,5,6)$.

Oltre a queste caratteristiche di migliore sensibilità analitica e clinica, il test combinato da noi valutato ha mostrato anche una buona capacità di discriminazione su campioni problematici. Infatti 17 dei 18 pazienti con false reattività ad un test anticorpale sono stati classificati correttamente come negativi con il test Architect Combo, e per contro tutti e 20 i pazienti classificati come positivi per HIV erano reattivi con il test Architect. $\mathrm{La}$ positività in Architect, in tutti e 4 bambini, di cui 3 in fase di negativizzazione e 1 positivo mette in evidenza la possibilità di questo test di rilevare anche gli anticorpi materni durante la fase di negativizzazione; in questi casi il dosaggio separato di anticorpi specifici e Ag p24 permette di dirimere la differenza. Infine, da un punto di vista strettamente analitico, la buona ripetibilità del test, sia sui controlli interni che su un campione di controllo di qualità interno, e la distribuzione dei campioni negativi (media segnale/valore soglia $0,120 \pm 0,050$, mediana 0,120 con 17,6 deviazioni standard tra la media dei negativi ed il valore soglia) garantiscono la robustezza del dosaggio e la consistenza dei risultati, soprattutto in termini di specificità.

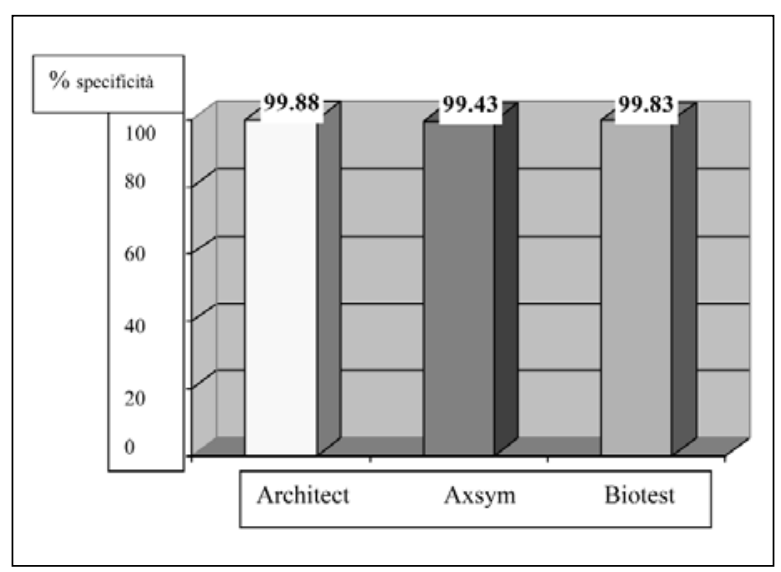

Figura I. Confronto della specificità dei tre test sierologici HIV valutati nel corso dello studio

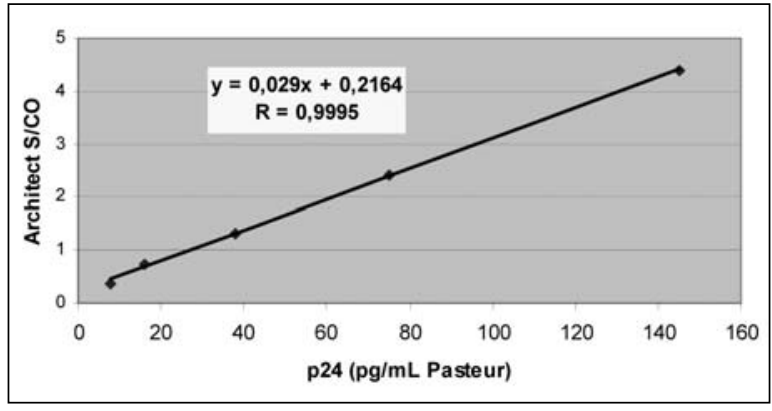

Figura II. Valutazione della sensibilità e linearità per antigene HIV p24 del test Architect HIV Combo su pannello BBI PRA 80 I HIV p24. La sensibilità, valutata mediante regressione lineare, è risultata pari a $22 \mathrm{pg} / \mathrm{mL}$

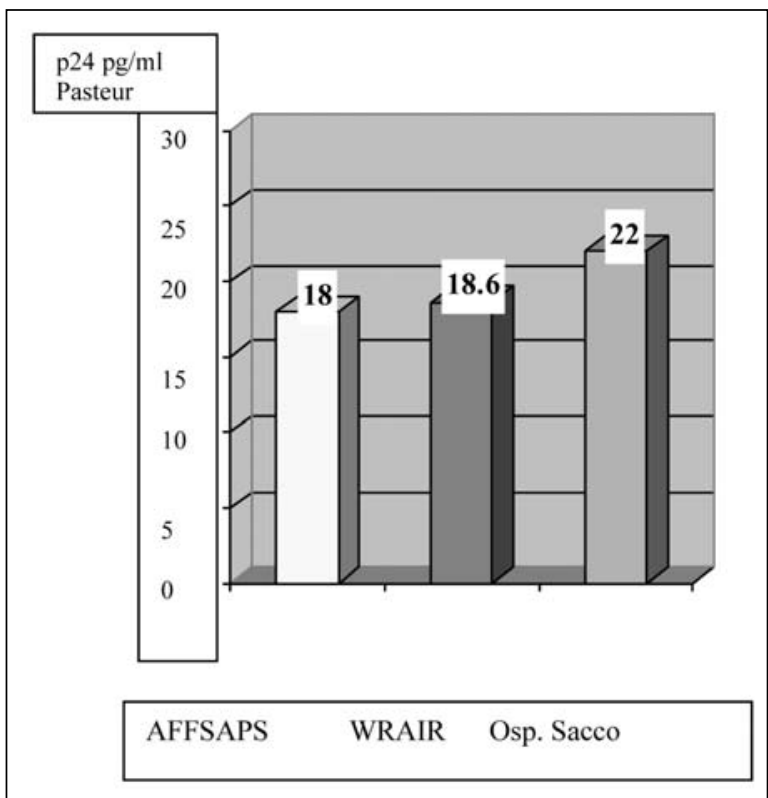

Figura III. Sensibilità per HIV Ag p24 del test Architect HIV Ag/Ab Combo: risultati su tre diversi pannelli

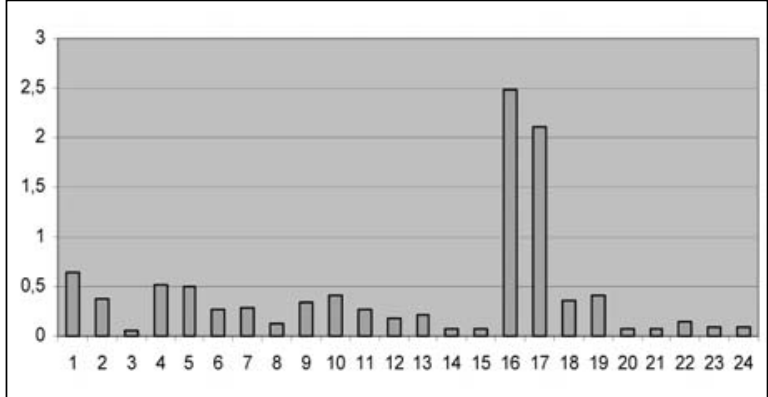

Figura IV. Segnale generato con il test Architect HIV Ag/Ab Combo su 24 campioni (da 18 pazienti) "falsi positivi" ad un test di screening anti-HIV di terza generazione. I valori sono espressi come segnale in rapporto al valore soglia (S/CO). Gli unici due campioni reattivi con Architect (S/CO 2,5 e 2,I) sono dello stesso paziente. 


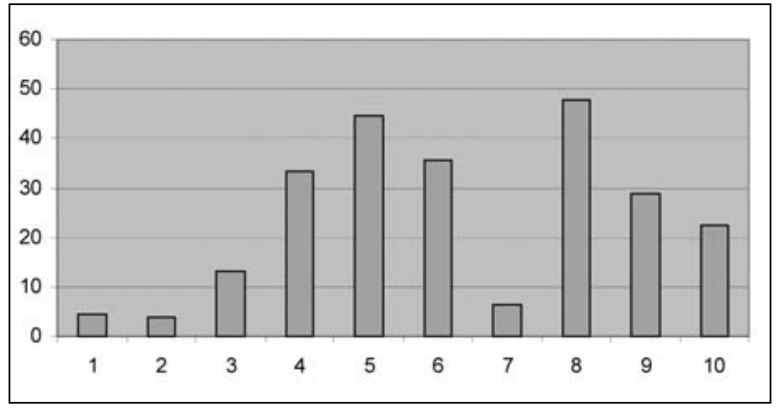

Figura V. Segnale generato con il test Architect $\mathrm{HIV} \mathrm{Ag/Ab}$ Combo su 10 campioni di sieroteca positivi per HIV e con risultati contrastanti tra test di primo e di secondo livello. I valori sono espressi come segnale in rapporto al valore soglia (S/CO), il segnale più basso ottenuto con Architect è oltre 3 volte la soglia.

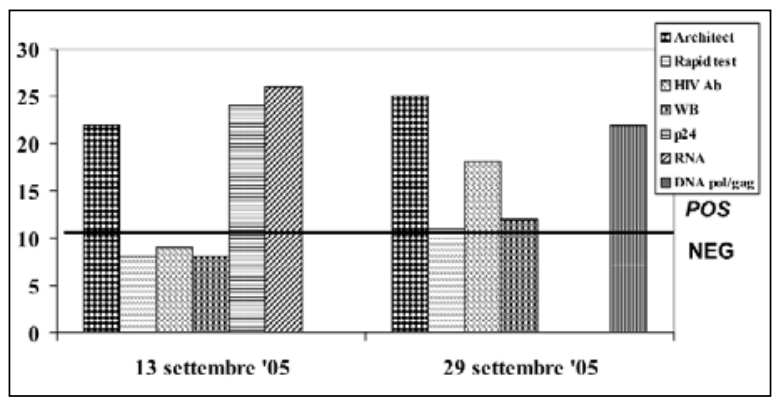

Figura VI. Sieroconversione HIV, caso n. I. Positività iniziale solo con il test combinato Architect, negatività con un test rapido e un test di terza generazione per soli anticorpi. I test virologici (antigene p24, HIV-RNA) sono positivi già sul primo prelievo. Dopo 16 giorni positività anche ai test di screening per anticorpi, ma senza conferma in WB (presenza di soli anti-p24).

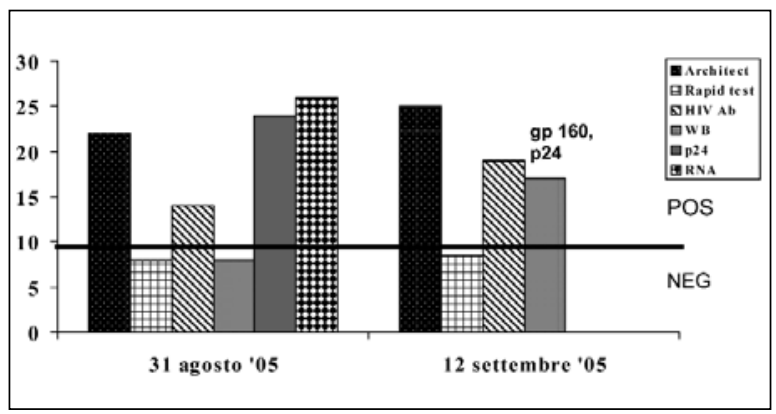

Figura VII. Sieroconversione HIV, caso n. 2. II primo prelievo era positivo sia con il test Architect HIV Combo che con un test per soli anticorpi, ma con WB negativo, mentre, come nel caso precedente, erano fortemente positivi sia l'antigene p24 che I'HIVRNA. A distanza di 12 giorni, oltre alla positività con i due test di screening, era indeterminato in WB (anti-gp/60 e anti-p24).

\section{BIBLIOGRAFIA}

1. (no authors listed) Time from HIV-1 seroconversion to AIDS and death before widespread use of highlyactive antiretroviral therapy: a collaborative re-analysis. Collaborative Group on AIDS Incubation and HIV Survival including the CASCADE EU Concerted Action on SeroConversion to AIDS and Death in Europe. Lancet 2000, 355: 1131-7.

2. Andersson S, Asjo B, Jenum PA, Manner I, et al. Relevance of a combined HIV antigen/ antibody assay to detect early HIV infections in a low prevalence population: case reports. Clin Lab 2004; 50: 109-13.

3. Fiebig EW, Wright DJ, Rawal BD, Garrett PE, Schimacher RT, Peddada L, Heldebrant C, Smith R, Conrad A, Kleinman SH, Busch MP. Dynamics of HIV viremia and antibody seroconversion in plasma donors: implications for diagnosis and staging of primary HIV infection. AIDS 2003; 17: 1871-9.

4. Guertler L, Muhlbacher A, Michl U, Hofmann H, et al. Reduction of the diagnostic window with a new combined p24 antigen and human immunodeficiency virus antibody-screening assay. J Virol Methods 1988, 75: 27-38.

5. Ly TD, Martin L, Daghfal D, Sandridge A, West D, et al. Seven Human Immunodeficiency Virus (HIV) Antigen-Antibody Combination Assays: Evaluation of HIV Seroconversion Sensitivity and Subtype Detection. J Clin Microbiol 2001, 39: 3122-8.

6. Sickinger E, Stieler M, Kaufman B, Kapprell HP, et al. Multicentre evaluation of a New Automated Enzyme-Linked Immunoassay for detection of Human Immunodeficiency Virus-Specific Antibodies and Antigen. J Clin Microbiol 2004, 42: 21-9.

7. Suligoi B, Pavoni N, Borghi V, et al. Epidemiologia dell'infezione da HIV in Italia. Epid Prev 2003, 27 : 73-9.

8. Suligoi B, Boros S, Camoni L, Lepore D, Ferri M, Roazzi P. Aggiornamento dei casi di AIDS notificato in Italia al 30 giugno 2005. Notiziario ISS 2005; 18, 11 (suppl.1)

9. Weber B. Fourth generation human immunodeficiency virus (HIV) screening assays with an improved sensitivity for p24 antigen close the second diagnostic window in primary HIV infection. J Clin Virol 2002; 25: 357-9.

\section{Nadia Zanchetta}

U. O. Microbiologia Azienda Ospedaliera

Polo Universitario "Luigi Sacco"

Via G. B. Grassi 74 - 20257 Milano

Te. 0239042479 - Fax 0239042313

E-mail: viro.microbiologia@hsacco.it 